\title{
The Role of Diagnostic Hysteroscopy in the Evaluation of Fallopian Tube Patency: a Short Review
}

\author{
Bedeutung der diagnostischen Hysteroskopie für \\ die Eileiterdurchgängigkeitsprüfung: eine kurze Übersicht
}

(c) (1) $\ominus$

Authors

Marlene Hager ${ }^{1}$, Inga Malin Simek ${ }^{1}$, Regina Promberger ${ }^{1,2}$, Johannes Ott ${ }^{1}$

\section{Affiliations}

1 Department of Obstetrics and Gynecology, Clinical Division of Gynecologic Endocrinology and Reproductive Medicine, Medical University of Vienna, Vienna, Austria

2 Department of Gynecology, Hanusch Krankenhaus, Vienna, Austria

Key words

office hysteroscopy, imaging tubal status, tubal pathology, uterine diagnosis, selective Fallopian tube perturbation

\section{Schlüsselwörter}

ambulante Hysteroskopie, bildgebende Verfahren zur Bewertung des Tubenstatus, Eileiterpathologie, Diagnostik der Gebärmutter, selektive Chromopertubation der Eileiter

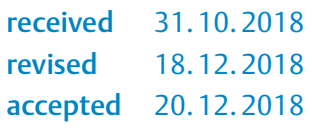

Bibliography

DOI https://doi.org/10.1055/a-0826-1326

Geburtsh Frauenheilk 2019; 79: 483-486 @ Georg Thieme Verlag KG Stuttgart · New York | ISSN 0016-5751

\section{Correspondence}

Johannes Ott, MD

Department of Obstetrics and Gynecology, Clinical Division of Gynecologic Endocrinology and Reproductive Medicine, Medical University of Vienna

Spitalgasse 23, 1090 Vienna, Austria

johannes.ott@meduniwien.ac.at

\section{ABSTRACT}

The relevance of diagnostic hysteroscopy, especially when performed in an outpatient "office" setting, in the evaluation process of infertility has increased within the last few years. Notably, several articles on a possible role for diagnostic hysteroscopy in the assessment of Fallopian tube patency have been published recently. Three relevant articles were identified. Visualizing a "flow effect" or air bubbles dispersing through the ostia, sonographically assessed shifts in cul de sac volume, and selective Fallopian tubal cannulation are reportedly promising hysteroscopic techniques. In this review, an overview of hysteroscopy, details about diagnostic reliability, and considerations with regard to ease of use and difficulties are summarized. Based on these articles, hysteroscopic evaluation of tubal patency seems to be a promising, clinically relevant field for future clinical research.

\section{ZUSAMMENFASSUNG}

Die Bedeutung der diagnostischen Hysteroskopie, besonders der ambulanten Hysteroskopie zur Abklärung von infertilität, hat in den letzten Jahren zugenommen. Vor Kurzem wurden mehrere Artikel über die potenzielle Rolle der diagnostischen Hysteroskopie für die Abklärung der Eileiterdurchgängigkeit veröffentlicht. Drei dieser Artikel sind für unsere Fragestellung relevant. Als vielversprechende hysteroskopische Methoden gelten die Visualisierung des „Durchflusses“ bzw. des Durchgangs von Luftbläschen durch das Ostium tubae, die mithilfe von Ultraschall untersuchten Veränderungen des DouglasRaums sowie die selektive Kanülierung des Eileiters. Dieser Übersichtsartikel fasst die wichtigsten Daten zur Hysteroskopie zusammen, vermittelt detaillierte Informationen über deren diagnostische Zuverlässigkeit und stellt Überlegungen zur Bedienbarkeit bzw. den damit assoziierten Schwierigkeiten an. Basierend auf diesen Artikeln stellt die hysteroskopische Bewertung der Eileiterdurchgängigkeit ein vielversprechendes, klinisch relevantes Feld für künftige klinische Forschungen dar. 
- Table 1 Overview about techniques of hysteroscopic assessment of Fallopian tube patency.

\begin{tabular}{|l|l|l|l|l|l|}
\hline Authors [reference] & Technique used & Outcome & Sample size & Sensitivity & Specificity \\
\hline Habibaj et al. [4] & $\begin{array}{l}\text { Pre- to post-hysteroscopic shifts } \\
\text { in cul-de-sac volume (sonography) }\end{array}$ & $\begin{array}{l}\text { Bilateral tubal } \\
\text { occlusion }\end{array}$ & 56 patients & $94.7 \%$ & $94.4 \%$ \\
\hline Parry et al. [8] & $\begin{array}{l}\text { Pre- to post-hysteroscopic shifts } \\
\text { in cul-de-sac volume (sonography) }\end{array}$ & $\begin{array}{l}\text { Bilateral tubal } \\
\text { occlusion }\end{array}$ & 89 patients & $92 \%$ \\
\hline Promberger et al. [7] & Hysteroscopic "flow" effect & Tubal occlusion & 998 tubes & $86.4 \%$ & - \\
\hline Parry et al. [8] & "Parryscope technique" with & Tubal occlusion & 170 tubes & $98.3-100 \%$ & $83.7 \%$ \\
\hline Török and Major [5] & Selective tubal pertubation & Tubal occlusion & 70 tubes & $83.3 \%$ \\
\hline
\end{tabular}

\section{Introduction}

Hysteroscopy, when combined with laparoscopy/chromopertubation, is considered the gold standard in the evaluation of tubal patency and the uterine cavity [1,2]. Together with ultrasound, it represents a first-line diagnostic tool for the evaluation of the uterine reproductive capacity. The high patient compliance rate, the few complications observed, and the possibility of direct tissue sampling are advantages [3].

There is increasing evidence that the possibility of performing hysteroscopy in an outpatient setting causes significantly less stress for patients as it is less invasive $[4,5]$ and is lower in cost for the healthcare system [5]. Thus, outpatient "office" diagnostic hysteroscopy has gained more importance in the course of the diagnostic evaluation of infertility and has been claimed to be the gold standard for evaluation of uterine-dependent infertility, since the patient can be offered low-risk diagnostic and treatment procedures that include targeted biopsy sampling, polypectomy, removal of submucous leiomyomas, lysis of synechiae, removal of retained conception products, metroplasty, and retrieval of dislodged intrauterine devices or foreign bodies [6]. In the last few years, several studies have reported that diagnostic hysteroscopy could also play a possible role in the assessment of tubal patency, which would extend its relevance even more. If a hysteroscopy is performed in a subfertile patient, it seems of interest to maximize the information, even on tubal patency. Thus, the aim of this narrative review on hysteroscopic Fallopian tube evaluation is to provide an overview of the different technical approaches published and their reliability.

\section{Review}

\section{Methods}

We conducted a review of literature electronically using PubMed, Cochrane Central Register of Controlled Trials, and Google Scholar to find studies on hysteroscopic Fallopian tube evaluation published from January 1980 to December 2017. The literature search was performed using the following search terms: "imaging tubal status"; "tubal pathology AND hysteroscopy"; "tubal patency AND hysteroscopy"; "office hysteroscopy"; "role of hysteroscopy AND diagnostic AND infertile"; and "hysteroscopy AND uterine diagnosis". Reference lists from the relevant publications were searched for additional studies on the subject. The studies were screened by title and abstract, and if after the reading of full text they met the inclusion criteria, they were selected by two authors $(\mathrm{MH}, \mathrm{JO})$. Inclusion criteria encompassed articles in the English language with the primary topic being diagnostic accuracy of hysteroscopic methods for evaluation of Fallopian tube patency. Four relevant articles were identified $[4,5,7,8]$. See $>$ Table 1 for an overview about reviewed studies.

\section{The value of hysteroscopic visualization of the Fallopian tube ostia}

In a recent retrospective study [7], we reviewed our experience with the flow of hysteroscopic fluid at the tubal ostia during hysteroscopy. The findings were compared with subsequently performed laparoscopic chromopertubation. A positive hysteroscopic flow was defined as "the observation of either a swirl of the saline toward the ostia or saline directly traversing the ostia". Using this criterion, the absence of flow would be suggestive for any kind of tubal occlusion, either proximal or distal, whereas the presence of flow would indicate patency. The positive (PPV) and negative predictive values (NPV) for proximal tubal occlusion were 92.9\% (90.7-94.7) and 64.3\% (95\% Cl: 58.3-69.9; $\mathrm{p}<0.001)$, respectively. PPV and NPV for distal tubal occlusion were $98.1 \%$ (95\% Cl: 96.8-99.0) and 23.3\% (95\% Cl: 16.3-31.5; $\mathrm{p}<0.001)$, respectively. Despite the large number of evaluated tubes (998), this study was limited by its retrospective design [7].

A comparable technique was used in a recently published prospective study. Parry et al. evaluated the accuracy of an air-bubbling ("Parryscope technique") effect during the flow of the hysteroscopic fluid through the ostia, provoked by an air-infusion into the uterine cavity [8]. The technique enabled the researchers to provide an even more precise statement about tubal patency. In detail, 435 patients who underwent office hysteroscopy were included in the study and, for 89 of these patients, surgical data from consecutive laparoscopy were available. First, assessment of the uterine cavity, which had to last for at least ten seconds, was performed, in order to allow pressure equilibration before evaluation of the air-bubbling effect. Then, a small amount of air (approximately $0.25 \mathrm{ml}$ ) was infused through the flexible hysteroscope. The air entering the uterine cavity formed either one large air bubble or a stream of air bubbles going through the ostia, which was considered an indicator of tubal patency. In contrast, if there was only one small $(<2 \mathrm{~mm})$ air bubble entering the osti- 
um, or if air bubbles entered only before the pressure equilibration had finished, this was not considered an indicator of tubal patency. If air bubbles did not rapidly go through the ostia, they were observed for another 40 to 60 seconds. This was thought to allow differentiation of occlusion from transient spasm. For the comparison between hysteroscopic and laparoscopic findings, data from 170 tubes were available. The Parryscope technique had a sensitivity of $98.3 \%$ and a specificity ranging from 69.5 to $83.7 \%$ for tubal patency. The latter depended on the subjective amount of force exerted during chromopertubation [8]. This seems of additional interest, since

1. low pressure may better reflect physiologic conditions and

2. high pressure patency in previous research has been shown to result in lower spontaneous fecundity, at least in hysterosalpingography [9].

\section{Shifts in cul de sac volume}

The above-described study by Parry et al. [8] evaluated an additional approach: a transvaginal sonography was performed to evaluate the cul de sac fluid before and after the office hysteroscopy. The vast majority of women, i.e., $92 \%$, with bilateral tubal occlusion, as suspected by office hysteroscopy, had no meaningful shift in cul de sac fluid from pre- to post-hysteroscopy. Moreover, it became evident that the remaining $8 \%$ of women with bilateral occlusion, who did have an increase in cul de sac fluid, all had initially elevated cul de sac fluid volume. The authors assumed that the variation in the repeated observation might have accounted for the difference. Notably, the findings were comparable to bilateral patency at laparoscopy. There was a significantly lower rate of cul de sac fluid accumulation during hysteroscopy for those patients who had bilateral occlusion in consecutive laparoscopy [8]. Noteworthy, it had already been reported previously, that transvaginal sonography performed directly after diagnostic hysteroscopy would provide additional information regarding tubal patency [4].

\section{Selective Fallopian tube pertubation}

Török and Major published the results of a prospective study on the accuracy of tubal patency evaluation using selective pertubation during office hysteroscopy. These findings were also compared with laparoscopy [5]. They first performed basic office hysteroscopy, in which data about deformations of the uterine cavity and the endometrium were recorded. Then, a plastic catheter was inserted through a working channel on the hysteroscope. The tip of the catheter was placed directly at the tubal ostium and 2$10 \mathrm{ml}$ of methylene blue dye were injected. The idea was that patent tubes would allow the dye to pass and no blue fluid should be seen in the uterine cavity. In contrast, occluded tubes would not allow the dye to pass through and, thus, the uterine cavity would turn blue because of the backflow. After evaluation of the tubes via hysteroscopy, laparoscopy that included chromopertubation was performed. The prospective study included 70 tubes of 35 women. Notably, tubal patency could be predicted with a PPV of $87.5 \%$ and an NPV of $76.7 \%$ (overall accuracy $82.9 \%$ ) [5].

\section{Complications and technical difficulties of the techniques described}

It seems mandatory to mention that all of these techniques likely require experience and, thus, some kind of training should be necessary before a high diagnostic accuracy can be achieved. It has already been reported that there is a learning curve for hysteroscopy, at least for residents under teaching conditions $[10,11]$. However, in the study of Parry et al. [8], junior residents, after pelvic simulation training, were able to perform testing as efficient or at least as fast as senior hysteroscopists. Nonetheless, one might be concerned about learning issues, especially if new diagnostic criteria would have to be applied in an already well-mastered technique or new additional steps would have to be implemented into such a process.

Moreover, we believe that, in the course of office hysteroscopy, the patient's acceptance of a technique will be higher if it is less invasive, due to the shorter time needed and probably less handling-associated pain. Assuming this, visualization of the Fallopian tube ostia and sonographically assessed perioperative shifts in cul de sac volume $[7,8]$ might be tolerated more by patients than selective Fallopian tube perturbation [5]. However, one cannot assume similar diagnostic reliability for these procedures. It should be emphasized that Török and Major already suggested that their technique of office hysteroscopy-guided selective chromopertubation might be even more exact than laparoscopic chromopertubation because of the direct injection of the dye [5]. Thus, studies that would compare the effectiveness and side effects of these techniques will be needed in the future.

Moreover, despite the high accuracy values, techniques utilizing visualization of the Fallopian tube ostia $[7,8]$ might be affected by another technical difficulty: Promberger et al. mentioned that if the Fallopian tubes come into contact with cool sodium chloride solution, especially before laparoscopic chromopertubation, the tubes might tend to spasm, which would then result in a higher rate of tubal occlusions during chromopertubation, and therefore, also a higher rate of false-positive hysteroscopic "flow" results [7]. Notably, the recent video article of Parry et al. [12] directly addressed tubal spasm by noting that it was actually observed using the "Parryscope" technique. Moreover, one could also argue that in fact pain may drive spasm as much as or even more than saline temperature. In this case, the use of small calibre, flexible hysteroscopes without high pressure uterine distention would contribute to diagnostic accuracy.

What also needs to be addressed in future studies is the impact of high pressure needed to achieve Fallopian tube patency during chromopertubation. In other words, supraphysiologic pressures during chromopertubation under operating-room conditions and standard chromopertubation pressures [8] might be of importance for a woman's fertility. Notably, high pressure patency has been associated with lower pregnancy rates $[9,13]$. However, most studies on how infusion pressures at chromopertubation affect fecundity predate modern laparoscopy and should be re-explored [13]. Thus, laparoscopic chromopertubation, which serves as the gold standard for other techniques designed to test Fallopian tube patency, might also be biased by several influencing factors that will need to be clarified. 


\section{Conclusions}

All of the aforementioned techniques are quite promising. The direct visualization of flow does not need any additional effort, which is definitely an advantage of this technique, whose success likely depends on the experience of the conductor. Observing a shift in cul de sac volume is also a technique that is easy to perform, as only a vaginal ultrasound is needed to confirm an eventual increase in fluid. The disadvantage of this technique is that only bilateral tubal occlusions can be detected with high accuracy. Moreover, existing data have already suggested the need for prehysteroscopic ultrasound in order to identify patients with pre-existing cul de sac fluid, since these seem to have an increased risk for a false-positive sonographic result.

However, larger prospective studies are necessary to draw final conclusions on the feasibility, pitfalls, and the accuracy of all these techniques. As shown by Promberger et al., an analysis of which conditions increase a patient's risk for either false-negative or false-positive results would be of clinical interest [7]. The approach of Parry et al. to wait and extend the observation period in case of a negative result in order to distinguish between Fallopian tube spasms and real occlusions seems also of great interest and should be kept in mind for future studies [8]. Moreover, as a prerequisite for all further studies on tubal patency, the role of the need for high pressure to achieve a positive chromopertubation result must be clarified.

Despite all enthusiasm about these possible future options, one might be concerned about a few more limitations: there are differences between office hysteroscopy and standard diagnostic hysteroscopy. For example, in the study of Promberger et al., hysteroscopes with a larger diameter were used [7]. Moreover, it might be of influence whether the procedure would be performed under general anaesthesia or at least sedation or not. Without any kind of sedation, higher pressures as also used in chromopertubation would not be feasible. On the other hand, the need for sedation would make the method less attractive as an office based tool. In addition, it needs to be emphasized that office hysteroscopy would have to be accompanied by ultrasound, since the first does not offer information on the uterine wall or and on what happens outside the uterus, namely adhesions, hydrosalpinges etc. Thus, hysteroscopy alone will not be capable to replace the more invasive diagnostic procedure of laparoscopy, if other abnormalities are suspected and to prove/rule out endometriosis. In other words, the question remains, how important hysteroscopy without laparoscopy in infertility patients would be. All in all, we might be talking more about maximizing the informative output of diagnostic hysteroscopy for those patients who do not undergo concomitant laparoscopy.
Moreover, it would be desirable to have studies that would compare the various techniques. Hysteroscopic evaluation of tubal patency seems to be a promising field for future clinical research, and the questions to be addressed in future studies include: which technique provides the most accurate information about tubal patency and which technique is the best for an individual patient.

\section{Conflict of Interest}

The authors declare that they have no conflict of interest.

\section{References}

[1] Luciano DE, Exacoustos C, Luciano AA. Contrast ultrasonography for tubal patency. J Minim Invasive Gynecol 2014; 21: 994-998

[2] Godinjak Z, Idrizbegović E. Should diagnostic hysteroscopy be a routine procedure during diagnostic laparoscopy in infertile women? Bosn J Basic Med Sci 2008; 8: 44-47

[3] Campo R, Meier R, Dhont $\mathrm{N}$ et al. Implementation of hysteroscopy in an infertility clinic: The one-stop uterine diagnosis and treatment. Facts Views Vis Obgyn 2014; 6: 235-239

[4] Habibaj J, Kosova H, Bilali S et al. Comparison between transvaginal sonography after diagnostic hysteroscopy and laparoscopic chromopertubation for the assessment of tubal patency in infertile women. J Clin UItrasound 2012; 40: 68-73

[5] Török P, Major T. Accuracy of assessment of tubal patency with selective pertubation at office hysteroscopy compared with laparoscopy in infertile women. J Minim Invasive Gynecol 2012; 19: 627-630

[6] Salazar CA, Isaacson K. Office Operative Hysteroscopy - an Update. J Minim Invasive Gynecol 2018; 25: 199-208

[7] Promberger R, Simek IM, Nouri K et al. Accuracy of tubal patency assessment in diagnostic hysteroscopy compared to laparoscopy in infertile women: a retrospective cohort study. J Minim Invasive Gynecol 2018; 25: 794-799

[8] Parry JP, Richie D, Aldred J et al. Proximal tubal patency demonstrated through air infusion during flexible office hysteroscopy is predictive of whole tubal patency. J Minim Invasive Gynecol 2017; 24: 646-652

[9] Totani R. The value of high-pressure hysterosalpingography with new cannula. Reproduccion 1982; 6: 167-177

[10] Issat T, Beta J, Januszewski M et al. Cumulative summation test for learning curve (LC-CUSUM) in outpatient hysteroscopy. Ginekol Pol 2017; 88: 9-12

[11] Elessawy M, Skrzipczyk M, Eckmann-Scholz C et al. Integration and Validation of Hysteroscopy Simulation in the Surgical Training Curriculum. J Surg Educ 2014; 74: 84-90

[12] Parry JP, Riche D, Rushing J et al. Performing the Parryscope technique gently for office tubal patency assessment. Fertil Steril 2017; 108: 718

[13] Karande CV, Pratt ED, Gleicher N. The assessment of tubal functional status by tubal perfusion pressure measurements. Hum Reprod Update 1996; 2: 429-433 